\title{
Chest Wall Necrosis, CTCAE
}

National Cancer Institute

\section{Source}

National Cancer Institute. Chest Wall Necrosis, CT CAE. NCI Thesaurus. Code C143223.

A disorder characterized by a necrotic process occurring in the soft tissues of the chest wall including breast. 